\title{
Predictive Value of Radiographic Measurements as a Diagnostic Marker of Systolic Heart Failure in a Retrospective Study: An Old Method, a New Approach
}

\author{
Tolga Aksu, ${ }^{1,}$ Tumer Erdem Guler, ${ }^{1}$ Veli Kaya, ${ }^{2}$ Nurcan Arat, ${ }^{3}$ and Omac Tufekcioglu ${ }^{4}$ \\ ${ }^{1}$ Cardiology Department, Derince Training and Research Hospital, University of Health Sciences, Kocaeli, Turkey \\ ${ }^{2}$ Cardiology Department, Mersin State Hospital, Mersin, Turkey \\ ${ }^{3}$ Cardiology Department, Istanbul Bilgi University, Faculty of Medicine, Istanbul, Turkey \\ ${ }^{4}$ Cardiology Department, Turkiye Yuksek Ihtisas Education and Research Hospital, Ankara, Turkey \\ "Corresponding author: Tolga Aksu, Cardiology Department, Derince Training and Research Hospital, University of Health Sciences, Kocaeli, Turkey. Tel: +90-5319903278, Fax: \\ +90-2623178000, E-mail: aksutolga@gmail.com
}

Received 2014 November 18; Revised 2014 December 13; Accepted 2014 December 27.

\begin{abstract}
Background: A number of earlier studies have attempted to establish the validity of various radiological parameters for the assessment of cardiac enlargement, but these were not fully quantitative as echocardiographic measurements.

Objectives: In our study, we aimed to determine the diagnostic accuracy of certain radiological parameters including the cardiothoracic ratio (CTR), cardiac area (CA) and cardiac volume (CV), derived from posteroanterior (PA) and lateral chest X-rays (CXR) in patients with impaired left ventricular ejection fraction (LVEF) suffering from dyspnea by comparing them with echocardiographic measurements of left ventricular (LV) dimensions and LVEF.

Patients and Methods: This retrospective study included 374 patients (258 females and 116 males) aged 35 to 78 years with the complaint of dyspnea. CXR and echocardiographic examination were performed on each patient upon admission. Based on LVEF, the patients were divided into two groups: Group 1 consisted of patients with impaired LVEF $(<50 \%)$ and group 2 consisted of patients with normal LVEF ( $\geq 50 \%$ ). The sensitivity, specificity, and cut-off points were evaluated by both receiver-operator characteristic (ROC) analyses and area under the ROC curve (AUC) to determine the diagnostic accuracy of CA, CV, and CTR.

Results: There was no significant relationship between CTR and LVEF, but CA and CV showed a strong correlation with LVEF $(\mathrm{P}<0.001$ and $\mathrm{P}<0.00001$, respectively). LV dimensions correlated better with CV than with $\mathrm{CA}(\mathrm{P}<0.00001$ and $\mathrm{P}<0.0001$, respectively). According to the analysis of ROC curves, the best cut-off value for CV for the diagnosis of systolic heart failure was $825 \mathrm{~mL}$.

Conclusion: The CV value correlated more closely with low LVEF and more accurately indicated an enlarged heart than CA or CTR. This may be due to the fact that an anteroposterior measurement of heart size is included in the former but not the latter two measurements. These study results may help quantify left heart enlargement with greater accuracy than may be obtained from standard CXR.
\end{abstract}

Keywords: Mass Chest X-Ray, Ejection Fraction, Ventricular

\section{Background}

Dyspnea is a frequently encountered symptom in daily clinical practice, and differential diagnosis is the cornerstone in management of patients. Heart failure (HF) is one of the most common causes of dyspnea. Clinical examination alone is usually unreliable for differential diagnosis of dyspnea, and demonstrating objective evidence of left ventricular (LV) dysfunction is crucial to correctly diagnosing $\mathrm{HF}(1,2)$. Transthoracic echocardiography (TTE) is currently the most commonly used imaging modality for the diagnosis of $\mathrm{HF}$, and will remain so for the foreseeable future. It provides a good general assessment of LV function. However, it is limited in patients with poor acoustic windows, it requires geometric assumptions in quantifying global LV systolic function, and its ability to provide specific tissue characterization is modest. Cardiac magnetic resonance (CMR) imaging is a highly accurate and reproducible imaging technique for the assessment of left and right ventricular volumes and global function. Additional information can be obtained when CMR is used with a contrast agent (3).

However, TTE and CMR are not available in every hospital and cannot be applied by all physicians. European Society of Cardiology (ESC) guidelines for HF indicate that a chest X-ray (CXR) is of limited but common use in the diagnostic work-up of patients with suspected HF (4). It has been concluded that LV systolic dysfunction may be present without cardiomegaly on the CXR. The cardiothoracic ratio (CTR) has been used to determine heart size in relevant reports. However, measurement of the car- 
diac area (CA) and cardiac volume (CV) with a plain radiograph, which is an old and little known technique, may more accurately predict the size of the heart. We aimed to demonstrate the diagnostic accuracy of radiographic measurements that are easily available in general hospitals for quantifying LV function and LV dimensions.

We compared left ventricular end-diastolic diameter (LVEDD), left ventricular end-systolic diameter (LVESD), and left ventricular ejection fraction (LVEF) derived from TTE with radiographic indices derived from posteroanterior $(\mathrm{PA})$ and lateral CXR in patients presenting with dyspnea.

\section{Objectives}

In our study, we aimed to determine the diagnostic accuracy of certain radiological parameters including the cardiothoracic ratio (CTR), cardiac area (CA) and cardiac volume (CV), derived from posteroanterior (PA) and lateral CXR in patients with impaired left ventricular ejection fraction (LVEF) suffering from dyspnea by comparing them with echocardiographic measurements of left ventricular (LV) dimensions and LVEF.

\section{Patients and Methods}

\subsection{Patients}

This retrospective study group consisted of 374 (258 females and 116 males) untreated patients who attended the outpatient clinic of the Department of Cardiology at the Turkiye Yuksek Ihtisas Education and Research Hospital for the differential diagnosis of dyspnea. Each patient's ECG, CXR, and TTE recordings were analyzed, and recordings were reviewed for physical examination findings, initial clinical presentation, family history, past medical history, smoking status, and New York Heart Association (NYHA) functional class. Presence of HF was detected by TTE. Based on LVEF, the patients were divided into two groups: Group 1 consisted of patients with impaired LVEF $(<50 \%)$ and group 2 consisted of those with normal LVEF ( $\geq 50 \%$ ) in line with the recommendations of the relevant guidelines (4). The patients with moderate or severe valvular pathology or pericardial effusion were excluded. Echocardiographic images and plain radiographs were evaluated by two independent observers. All radiographic images were checked by a radiologist who was blinded to the clinical characteristics and echocardiographic data of the patients. A flowchart illustrating the selection of study patients is presented in Figure 1.

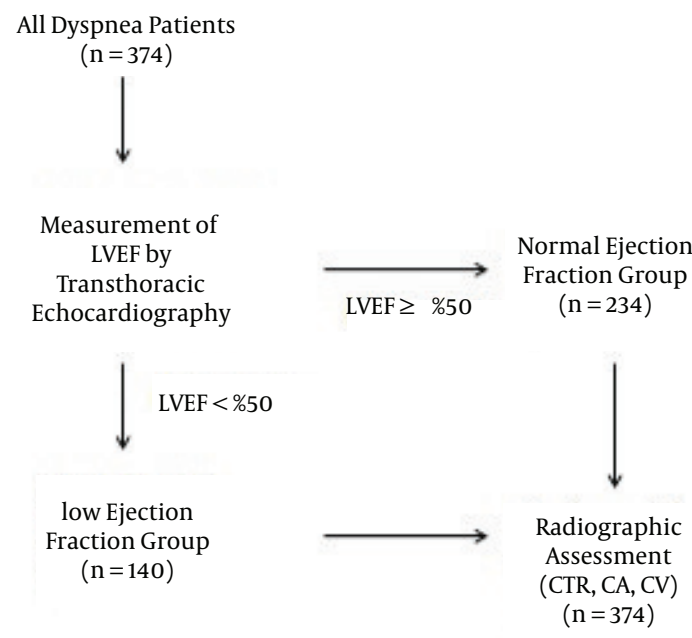

Figure 1. Flow chart illustrating the selection of patients; Abbreviations: CA, car diac area; CTR, cardiothoracic ratio; CV, cardiac volume; LVEF, left ventricular ejection fraction.

The protocol was approved by the ethical committee of the Turkiye Yuksek Ihtisas education and research hospital. Written consent was not obtained because of the retrospective design of the study.

\subsection{Radiographic Assessment}

The radiographic assessment was performed by a direct radiography (DR) system (Axiom Aristos, Siemens Medical Solutions, USA) in the radiology unit. The technicians were made blind to the results of the other investigations. CTR was evaluated solely via PA erect radiograph (Figure 2). No Antero-posterior (AP), supine, or seated films were accepted. CV was measured by using the method described by Keats and Enge (5) (Figure 2).

The length diameter $(\mathrm{L})$ is measured from the junction of the superior vena cava to the cardiac apex. The broad diameter (B) is taken from the junction of the right atrium and the diaphragm and the junction of the pulmonary artery. D represents the greatest horizontal diameter of the heart in lateral film. Thus, the calculation of $\mathrm{CV}$ requires both a PA and lateral film. $\mathrm{K}$ is a constant (0.63), and the value of $\mathrm{K}$ varies with different focal-film distances. In our study, the focal-film distance was $150 \mathrm{~cm}$ for a $\mathrm{K}$ value of 0.39. CA was calculated as described by Ungerleider and Gubner (6) (Equation 1):

$C A=B \times L \times\left(\frac{\pi}{4}\right)$ 

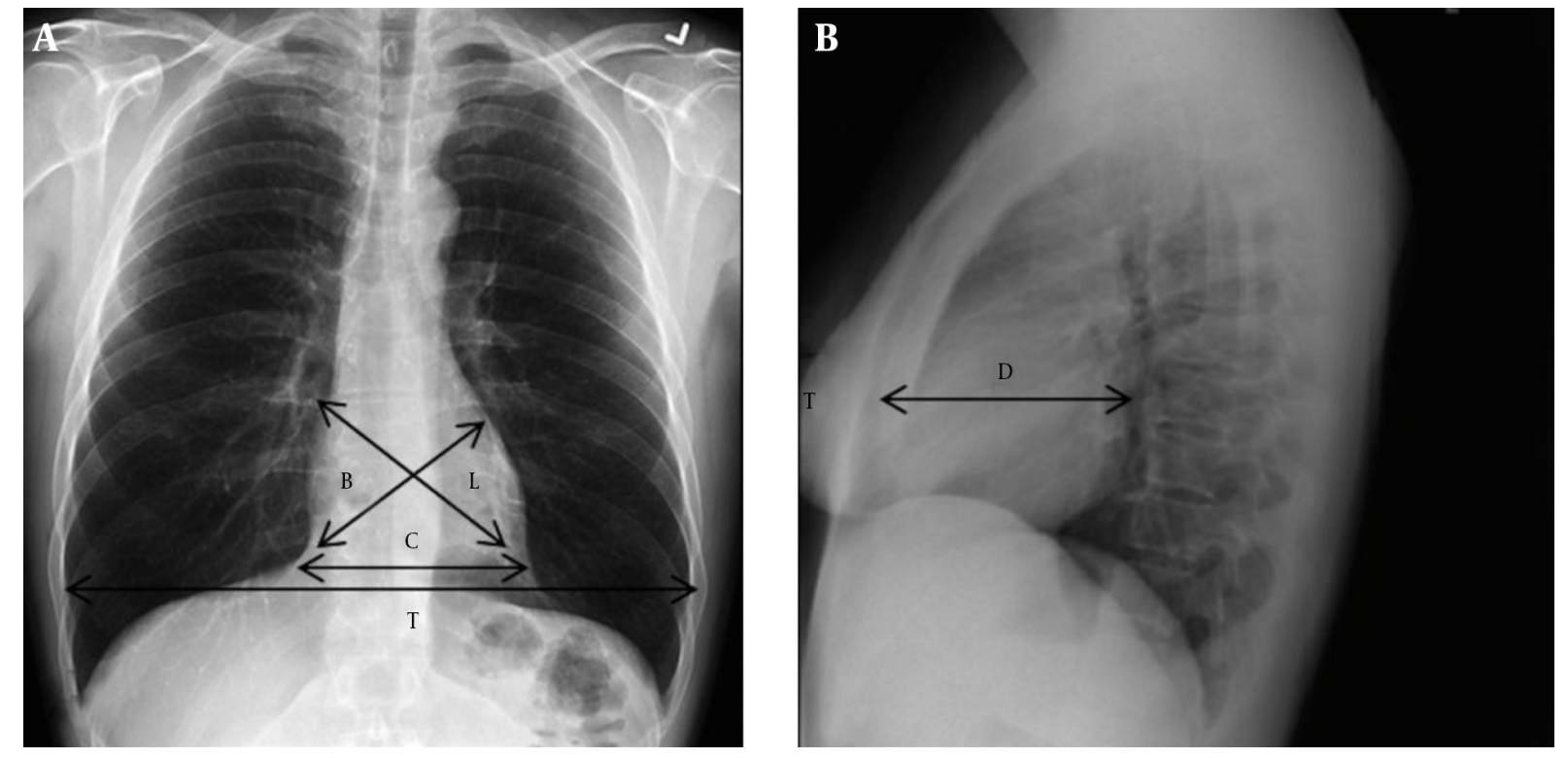

Figure 2. The measurements of cardiothoracic ratio, cardiac area, and cardiac volume are shown on posteroanterior (A) and lateral (B) chest X-Rays. B, from the right cardiophrenic angle to the base of the main pulmonary artery segment; $\mathrm{D}$, the greatest cardiac diameter on the lateral film; and $\mathrm{L}$, from the junction of the right atrium and superior vena cava to the apex. Abbreviations: $C A$, cardiac area; $C V$, cardiac volume: $C V=L \times B \times D \times K$.

\subsection{Echocardiographic Study}

A skilled echocardiographer blind to the clinical features of the patients performed the echocardiographic study using a Vivid 7 (GE Healthcare, Horten, Norway) ultrasound system. Basic measurements included LV diameters and LA diameters by 2D echocardiography with settings per recommendations by the American Society of Echocardiography (7). Left ventricular volumes and LVEF were calculated by using the biplane method (modified Simpson's rule) as recommended by the American Society of Echocardiography (8).

\subsection{Statistical Analysis}

Statistical analysis was performed with the SPSS/PC software package (version $\mathbf{1 5 . 0}$ for Windows; SPSS Inc., Chicago, Il, USA). Categorical data are reported as proportions, and continuous variables are expressed as mean \pm standard deviation. Frequency comparisons were made using the t-test, Kruskal-Wallis test, Mann-Whitney U test, and chi-square test as appropriate. Analyses of continuous variables were performed using the Student's t-test or analysis of variance (AVONA) for comparison of normally distributed data. In addition, the association between CXR and TTE-based parameters was assessed using the Spearman's rank correlation test. Receiver operating characteristic (ROC) curves were drawn to quantify the ability of CTR, CA, and CV to predict outcome and constructed to determine the cut-off value with the highest discriminating power. ROC curve analyses are graphically represented. For the cut point of $\mathrm{CV}$, sensitivity and specificity were reported. Statistical significance was set at a $\mathrm{P}<0.05$. All reported probability values were two-sided.

\section{Results}

The median age of the studied population was $59 \pm 12$ years with a male to female ratio of 0.31 . Demographic data are shown in Table 1. Group 1 consisted of 81 males and 59 females. In all groups, the mean CTR, CA, and CV values were $0.59 \pm 0.09,161 \pm 47 \mathrm{~cm}^{2}$, and $584 \pm 186 \mathrm{~mL}$, respectively. The CXR indices of group 1 and group 2 are represented in Table 2 . There was no significant relationship between CTR and LVEF. The relation between LVEF and various radiographic indices is shown in Table 3 and Figures 3 and 4.

According to the analysis of ROC curves, the best cutoff value for $\mathrm{CV}$ for the diagnosis of HF was $825 \mathrm{~mL}$. In the analysis of all patients, the area under the ROC curve of CV was 0.712 (Figure 5). The sensitivity and specificity of CV in the diagnosis of HF are shown in Table 4 and Figure 5.

\section{Discussion}

Dyspnea is a commonly seen problem in patients presenting to the emergency department. The etiology of 
Table 1. Clinical Demographic Data in Two Groups of Patients Determined Based on Left Ventricular Ejection Fraction ${ }^{a}$

\begin{tabular}{|c|c|c|c|c|}
\hline \multirow{2}{*}{ Demographics } & \multicolumn{2}{|l|}{ Group 1} & \multicolumn{2}{|l|}{ Group 2} \\
\hline & Number or Mean (SD) & Percent & Number or Mean (SD) & Percent \\
\hline NYHA I & 10 & 7 & 40 & 17 \\
\hline NYHA II & 68 & 49 & 106 & 45 \\
\hline NYHA III & 32 & 23 & 50 & 21 \\
\hline NYHA IV & 30 & 21 & 38 & 16 \\
\hline Orthopnea & 57 & 41 & 85 & 36 \\
\hline Angina $^{a}$ & 67 & 48 & 11 & 5 \\
\hline Pretibial edema & 20 & 14 & 54 & 23 \\
\hline Ascites & 10 & 7 & 38 & 16 \\
\hline Rales & 36 & 26 & 64 & 27 \\
\hline Height & $1.64(0.1)$ & & $1.66(0.1)$ & \\
\hline Weight & $83.3(14.8)$ & & $82.2(12.5)$ & \\
\hline
\end{tabular}

Abbreviation: NYHA, New York Heart Association.

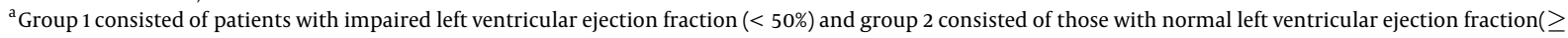
$50 \%)$.

Table 2. Plain Chest X-ray Parameters in Two Groups ${ }^{\mathrm{a}}$

\begin{tabular}{|c|c|c|c|c|c|c|c|c|}
\hline & \multicolumn{4}{|c|}{ Group 1} & \multicolumn{4}{|c|}{ Group 2} \\
\hline & Mean & Standard Deviation & Minimum & Maximum & Mean & Standard Deviation & Minimum & Maximum \\
\hline CTR & 0.60 & 0.06 & 0.44 & 0.78 & 0.56 & 0.09 & 0.31 & 0.64 \\
\hline $\mathrm{CA}^{\mathrm{a}}$ & 174 & 53 & 116 & 413 & 146 & 42 & 84 & 216 \\
\hline$C V^{b}$ & 667 & 223 & 186 & 1,408 & 485 & 206 & 252 & 733 \\
\hline
\end{tabular}

Abbreviations: CA, cardiac area; CTR, cardiothoracic ratio; CV, cardiac volume.

${ }^{a}$ Group 1 consisted of patients with impaired left ventricular ejection fraction $(<50 \%)$ and group 2 consisted of those with normal left ventricular ejection fraction( $\geq$ $50 \%)$.

${ }^{\mathrm{b}} \mathrm{P}<0.0001$.

${ }^{\mathrm{c}} \mathrm{P}<0.00001$

Table 3. Relations Between the Different Echocardiographic Measures of Left Ventricular Function and Chest X-ray Indices

\begin{tabular}{lcccccc}
\hline & LVEDD & & & LVESD & & LVEF \\
\cline { 2 - 7 } & Correlation Coefficient & PValue & Correlation Coefficient & PValue & Correlation Coefficient & P Value \\
\hline CTR & .031 & 0.344 & .062 & 0.059 & .208 \\
CA & .19 & $<0.0001$ & .19 & $<0.0001$ & .29 \\
CV & .36 & $<0.00001$ & .35 & $<0.00001$ & .37 \\
\hline
\end{tabular}

Abbreviations: CA, cardiac area; CTR, cardiothoracic ratio; CV, cardiac volume; LVEDD, left ventricular end-diastolic diameter; LVEF, left ventricular ejection fraction; LVESD, left ventricular end-systolic diameter.

dyspnea is usually challenging because many clinical conditions should be kept in mind in the differential diagnosis. Correct diagnosis, especially in situations that require rapid intervention, can be life saving. Cardiovascular causes of dyspnea are associated with severe morbidity and mortality $(9,10)$. Although history and physical exam- ination play an important role in the differential diagnosis of cardiovascular causes from the other etiologies, the symptoms are usually similar, and the gold standard for diagnosis is TTE. However, echocardiography devices and a physician who can evaluate the results of echocardiography are not available in many centers. 
Table 4. Sensitivity and Specificity and Receiver Operating Characteristics Analysis of X-ray Indices in the Diagnosis of Heart Failure

\begin{tabular}{|c|c|c|c|c|c|}
\hline Sample & ROC Curve Area & Standard Error & Cut Off & Sensitivity & Specificity \\
\hline CTR & 0.543 & 0.019 & NA & NA & NA \\
\hline CA & 0.577 & 0.019 & NA & NA & NA \\
\hline CV & 0.712 & 0.017 & $900 ; 550$ & $45 ; 95$ & $95 ; 40$ \\
\hline
\end{tabular}

Abbreviations: CA, cardiac area; CTR, cardiothoracic ratio; CV, cardiac volume; ROC, receiver operating characteristic; NA, not available.

A

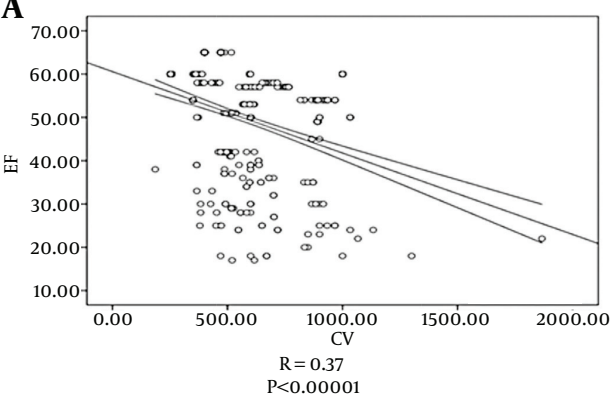

$\mathbf{B}$

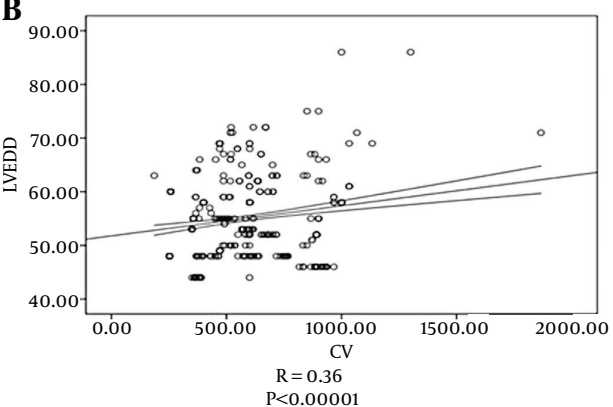

C

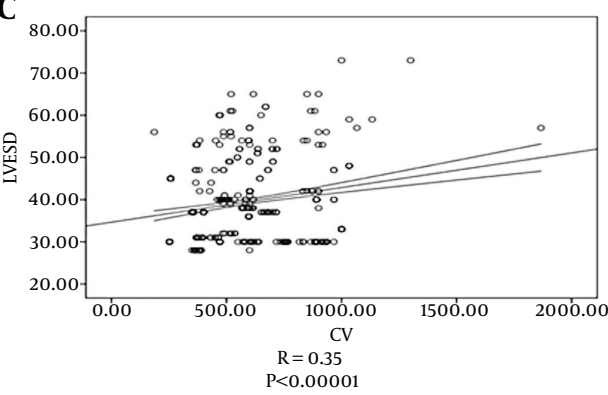

Figure 3. Correlation between echocardiographic parameters and cardiac volume Parameters are A, ejection fraction; B, left ventricular enddiastolic diameter, and C, left ventricular endsystolic diameter. Spearman's rank test showed a significant positive correlation between CV and LV dimensions and a significant negative correlation between CV and LVEF. Abbreviations: CV, cardiac volume; LVEF, left ventricular ejection fraction, LVEDD, left ventricular end-diastolic diameter; LVESD, left ventricular end-systolic diameter; EF, ejection fraction.

In contrast, direct radiographic study is available at almost all hospitals and does not require expertise in cardiology for the interpretation of the findings. Radiographic
A

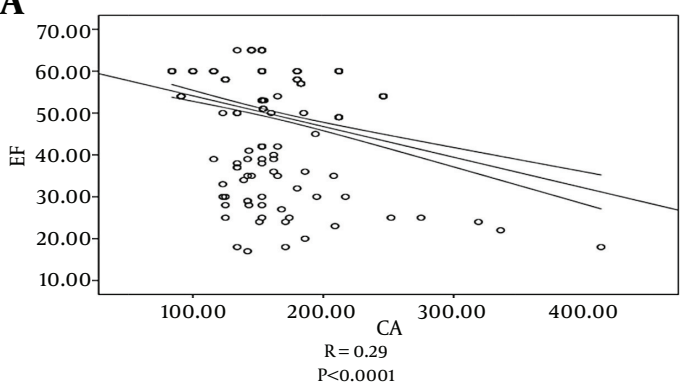

B

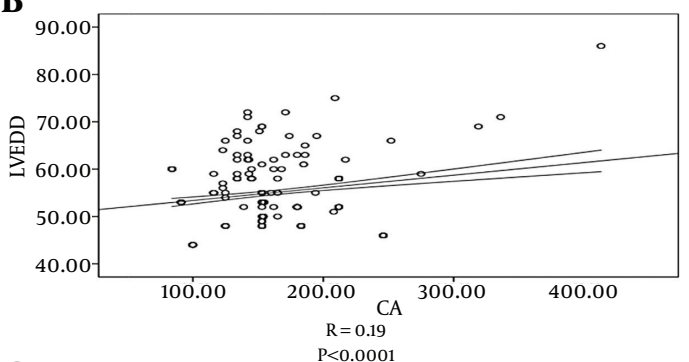

C

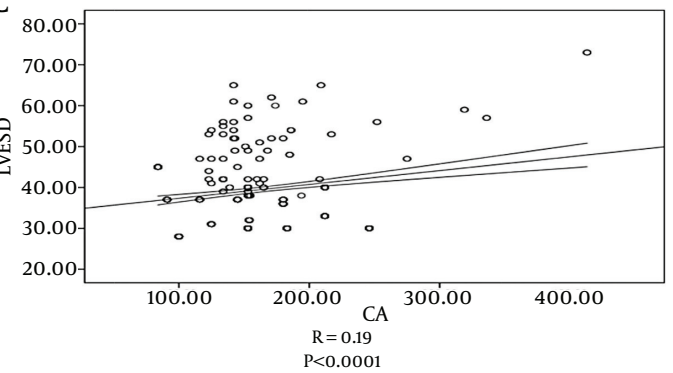

Figure 4. Correlation between echocardiographic parameters and cardiac area. Parameters are A, ejection fraction; B, left ventricular enddiastolic diameter, and C, left ventricular endsystolic diameter Spearman's rank test showed a moderately significant positive correlation between CA and LV dimensions and a significant negative correlation between CA and LVEF. Abbreviations: CA, cardiac area; LVEF, left ventricular ejection fraction; LVEDD, left ventricular end-diastolic diameter; LVESD, left ventricular end-systolic diameter; $\mathrm{EF}$, ejection fraction.

study is also cheaper and can be applied quickly. Several studies in adults have compared radiographic and angiographic data and have generally found a good correlation between radiographic CV's and various angiographic left heart measurements $(5,6,11)$. 


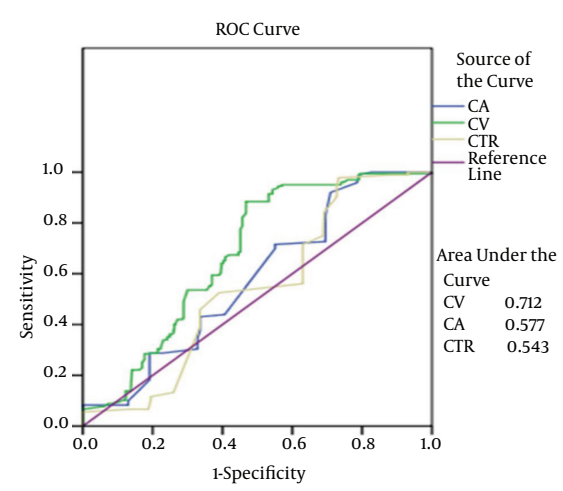

Figure 5. Receiver operating characteristic curve analysis; diagnostic performance of CV, CA, and CTR in the detection of systolic HF (LVEF < 50\%); SE: 0.05. Area under the receiver operating characteristic curve values of $\mathrm{CV}, \mathrm{CA}$, and CTR in systolic $\mathrm{HF}$ from the study; Abbreviations: CA, cardiac area; CTR, cardiothoracic ratio; CV, cardiac volume; LVEF, left ventricular ejection fraction.

There are contradictory results in the literature concerning the comparison of echocardiographic and radiological cardiac dimensions. Davidson et al. (12) demonstrated that although there was a significant correlation between the radiographic total CV and echocardiographic ventricular volumes, especially in left-sided pathologies, CTR and CA did not correlate well with echocardiographic measurements. However, Lewis (13) found a high degree of correlation between CA and LVEDV in patients with pure aortic valve insufficiency. In addition, Glover et al. (14) demonstrated that $\mathrm{CV}$ gives the greatest diagnostic accuracy for measurement of the left ventricular dimensions (79\%) when compared with CTR.

In our study, we found a strong relationship between echocardiographic dimensions and CA and CV in patients with dyspnea. Therefore, we believe that physicians may use CA and CV measurements in the differential diagnosis of patients presenting to emergency departments with dyspnea, which is a cheaper and more rapidly available diagnostic tool.

There is no absolute standard for the diagnosis of HF, although TTE is accepted as the gold standard. In this study, we aimed to define the potential role of CXR in the diagnosis of systolic HF and LV enlargement in outpatient cardiology clinics. However, the potential usage of CXR may be to identify patients requiring assessment by TTE in emergency departments. The patients in this study were diagnosed on the basis of symptoms compatible with HF in the presence of objective evidence of left ventricular dysfunction (LVEF < 50\%). Because this was a retrospective study, it is not possible to be certain of the standardization for chest radiography. Poor inhalation can cause an overestimated CTR. The wide scatter of the points suggests that this was not a systematic error.

In conclusion, $\mathrm{CV}$ on chest radiography may be a satisfactory, cheap, and easily available method for diagnosing cardiac enlargement or HF.

\subsection{Limitations}

This study was a retrospectively and cross-sectionally designed single center study, which means that statistical power of the correlation between echocardiograhic and radiographic parameters may be weaker. To overcome these limitations, the patients for whom echocardiographic and radiologic evaluations were performed at the same period were included in study. Another major limitation of this study is that we excluded patients with moderate or severe valvular pathology or pericardial effusion. It is well known that these conditions are common among HF patients, and excluding them will affect the results. In addition, the etiology of HF and diastolic HF were not taken into consideration because echocardiographic or radiological cardiac dimensions may vary due to underlying conditions. The cutoff point for LVEF (50\%) may be questionable. However, entirely normal ejection fraction is accepted as $>50 \%$ in the relevant guideline. Therefore, we used $50 \%$ as the cut-off point in our study.

\section{Acknowledgments}

We thank all of the cardiology staff at the Turkiye Yuksek Ihtisas Hospital.

\section{Footnotes}

Authors' Contribution: Tolga Aksu conceived of and designed this study and collected all patient data. Tumer Erdem Guler and Veli Kaya participated in the study design and performed the statistical analysis. Nurcan Arat and Omac Tufekcioglu drafted the manuscript and gave final approval of the version to be published. All authors read and approved the final manuscript.

Financial Disclosure: None declared.

Funding/Support: None declared.

\section{References}

1. Harlan WR, oberman A, Grimm R, Rosati RA. Chronic congestive heart failure in coronary artery disease: clinical criteria. Ann Intern Med. 1977;86(2):133-8. [PubMed: 835934].

2. Stevenson LW, Perloff JK. The limited reliability of physical signs for estimating hemodynamics in chronic heart failure. JAMA. 1989;261(6):884-8. [PubMed: 2913385]. 
3. Karamitsos TD, Francis JM, Myerson S, Selvanayagam JB, Neubauer $S$. The role of cardiovascular magnetic resonance imaging in heart failure. J Am Coll Cardiol. 2009;54(15):1407-24. doi: 10.1016/j.jacc.2009.04.094. [PubMed: 19796734].

4. McMurray JJ, Adamopoulos S, Anker SD, Auricchio A, Bohm M, Dickstein K, et al. ESC Guidelines for the diagnosis and treatment of acute and chronic heart failure 2012: The Task Force for the Diagnosis and Treatment of Acute and Chronic Heart Failure 2012 of the European Society of Cardiology. Developed in collaboration with the Heart Failure Association (HFA) of the ESC. Eur Heart J. 2012;33(14):1787-847. doi: 10.1093/eurheartj/ehs104. [PubMed: 22611136].

5. Keats TE, Enge IP. Cardiac mensuration by the cardiac volume method. Radiology. 1965;85(5):850-5. doi: 10.1148/85.5.850. [PubMed: 5844523].

6. Ungerleider HE, Gubner R. Evaluation of heart size measurements. American Heart Journal. 1942;24(4):494-510. doi: 10.1016/s00028703(42)90966-9.

7. Gottdiener JS, Bednarz J, Devereux R, Gardin J, Klein A, Manning WJ, et al. American Society of Echocardiography recommendations for use of echocardiography in clinical trials. J Am Soc Echocardiogr. 2004;17(10):1086-119. doi: 10.1016/j.echo.2004.07.013. [PubMed: 15452478].

8. Schiller NB, Shah PM, Crawford M, DeMaria A, Devereux R, Feigenbaum $\mathrm{H}$, et al. Recommendations for quantitation of the left ventricle by two-dimensional echocardiography. American Society of Echocardiography Committee on Standards, Subcommittee on Quantitation of Two-Dimensional Echocardiograms. J Am Soc Echocardiogr. 1989;2(5):358-67. [PubMed: 2698218].

9. Mahler DA, Mejia R. In: Dyspnea. Davis GS, editor. New York: Marcel Dekker;1999. pp. 221-32.

10. Kunitoh H, Watanabe K, Sajima Y. Clinical features to predict hypoxia and/or hypercapnia in acute asthma attacks. J Asthma. 1994;31(5):4017. [PubMed: 7928936].

11. Clark AL, Coats AJ. Unreliability of cardiothoracic ratio as a marker of left ventricular impairment: comparison with radionuclide ventriculography and echocardiography. Postgrad Med J. 2000;76(895):289-91. [PubMed: 10775282].

12. Davidson A, Krull F, Kallfelz HC. Cardiomegaly-what does it mean? A comparison of echocardiographic to radiological cardiac dimensions in children. Pediatr Cardiol. 1990;11(4):181-5. doi: 10.1007/BF02238363. [PubMed: 2148819].

13. Lewis RP, Bristow JD, Griswold HE. Radiographic heart size and left ventricular volume in aortic valve disease. Am JCardiol. 1971;27(3):2503. [PubMed: 5101316].

14. Glover L, Baxley WA, Dodge HT. A quantitative evaluation of heart size measurements from chest roentgenograms. Circulation. 1973;47(6):1289-96. [PubMed: 4267844]. 